\title{
Effect of correction of endothelial dysfunction on clinical and immunological parameters in patients with bronchial asthma
}

\begin{abstract}
Aim: The aim of the study was to study the effect of correction of endothelial dysfunction on the clinical and immunological parameters of patients with bronchial asthma with a long history of the disease.

Materials and methods: We examined 86 patients with mild and moderate-onset persistent asthma at the age of $18-55$ years (38.5 \pm 4.2 years), with a duration of disease more than 12 years (on average $16+4.5$ years). Two groups were formed by random sampling. Patients of the main group $(n=44)$, in addition to the standard basic therapy (GINA, 2007) received a nitric oxide donor - L-arginine. The comparison group $(n=42)$ received only basic therapy. As a control, 15 practically healthy persons were examined. The condition of the endothelial system was assessed before and after the ten-day course, and also after 1 month of treatment according to the following indices. In the dynamics of treatment, the immunological parameters in the blood and in the condensate of exhaled air were also evaluated.
\end{abstract}

Conclusion: In patients with a long course of persistent asthma of moderate severity with the use of L-arginine in addition to basic therapy, which contributes to an earlier and reliable improvement of the immunological parameters studied.

Keywords: bronchial asthma, endothelial system, interleukins, L-arginine
Research Article
Volume 5 Issue 2 - 2018

\section{Alyavi AL, Bazarova SA, Sadykova GA, Dzhambekova GS}

Republican Specialized Scientific and Practical Medical Center for Therapy and Medical Rehabilitation Tashkent, Uzbekistan

Correspondence: SA Bazarov, Republican Specialized Scientific and Practical Medical Center for Therapy and Medical Rehabilitation Tashkent, Uzbekistan, Tel +99894653।358, Email d-sayyorik@mail.ru

Received: December 27, 2017| Published: March 06, 2018

\section{Introduction}

Today, bronchial asthma (BA) remains relevant as a serious chronic respiratory disease. Despite the rapid development of the pharmaceutical industry, AD is a serious public health problem. ${ }^{1}$ Significant progress has been made in understanding the pathogenetic mechanisms of asthma. In recent years, international programs to combat asthma have been developed and implemented. ${ }^{2}$ One of the priority areas of modern medicine is the search for diagnostic and therapeutic approaches based on further study of the mechanisms of development and progression of asthma. At present, cardiovascular effects are considered as systemic manifestations of asthma, among which endothelial dysfunction appears as the primary link of the vessel wall damage. ${ }^{4}$ Numerous recent studies have established that activation and/or damage to the endothelium is of fundamental importance in the development of a wide range of pathological processes in the cardiovascular, nervous, endocrine systems, including in the respiratory system. ${ }^{2,5} \mathrm{~A}$ wide range of functions performed by certain endothelial factors presupposes the direct participation of these substances in the pathophysiological mechanisms of asthma. ${ }^{6,7}$ In the formation of functional and structural disorders in $\mathrm{AD}$, an important role is played by nitrogen oxide (NO), produced by various forms of NO-synthase (NOS). It has been shown that under physiological conditions, the picomolar amounts of NO synthesized by constitutive NOS exert bronchodilator, anti-inflammatory and antiproliferative actions, while under the conditions of inflammation, the supersynthesis of NO by the inducible isoform enzyme leads to the opposite effect. ${ }^{7-9}$ The immune system and the nitric oxide system is an interactive process. Immunocompetent cells and cytokines regulate the effects of expression of all types of nitrate synthases and, accordingly, the production of nitric oxide. In turn, the molecule of nitric oxide, as a "signal molecule of intercellular interaction" has a corrective effect on the function of the links of the immune system. The aim of the study was to study the effect of correction of endothelial dysfunction on the clinical and immunological parameters of patients with asthma with a long history of the disease.

\section{Materials and methods}

A total of 86 patients with moderate and moderate persistent asthma, aged 18 to 55 years ( $38.5 \pm 4.2$ years), with a disease duration of more than 12 years (mean $16+4.5$ years) were examined. Two groups were formed by random sampling. Patients of the main group $(n=44)$, in addition to the standard basic therapy (GINA, 2007) received a nitric oxide donor - L-arginine. The drug $(100 \mathrm{ml}$ of a $4.2 \%$ solution, Tivortin, Yuriyafarm, Ukraine) was injected iv by drop, once a day, for 10days. The comparison group $(n=42)$ received only basic therapy. As a control, 15 practically healthy persons were examined. The state of the endothelial system was assessed before and after the ten-day course, and also after 1 month of treatment, according to the following indices: a change in the level of the main stable metabolites of nitric oxide $\left(\mathrm{NO}_{2} / \mathrm{NO}_{3}\right)$, which were determined using the Griss reagent (PP Golikov et al. 2004) in the blood serum and in the condensate of exhaled air (KVV). The collection of KVV was carried out by the method of GI Sidorenko et al. ${ }^{10}$ modified by us. The determination of the level of L-arginine in plasma was carried out by horizontal chromatography on a thin layer of the sorbent. In the dynamics of treatment, the immunological parameters in the blood and in the KVV were also evaluated. The content of interleukin-4 (IL-4), interleukin-8 (IL-8), tumor necrosis factor- $\alpha$ (TNF- $\alpha$ ) in the blood serum and in HBV was determined by solid-phase enzyme-linked immunosorbent assay. Reagent kits for immunoenzymatic analysis of human cytokines from Vector-Best (Novosibirsk) were used. Statistical processing of data 
was carried out using the Microsoft Excel spreadsheet for Windows 2007. All data were recorded in summary tables, for arithmetic mean values, their standard deviations and standard errors $(\mathrm{M} \pm \mathrm{m})$ were calculated. The reliability of the difference between the groups was assessed with the calculation of the Student's test.

\section{Results}

The initial data showed that in both groups of patients the content of stable metabolites of nitric oxide was lower than normal values in blood and in CVI $(\mathrm{p}<0.05)$ (Figure 1), and the level of L-arginine in plasma was decreased $(p<0,05)$ (Figure 2). The initial data of immunological indices in patients of both groups were comparable $(p>0.05)$ and were characterized by a significant increase in the content of IL-4 and TNF- $\alpha$ in the blood serum and in the KVB patients. At the same time, the increase in IL- 8 was unreliable and was noted only in 12 patients. It is known that IL-8 belongs to the chemokine group in its properties, and is able to induce activation and chemotaxis of leukocytes in response to infection. Previously, it was found that IL-8 in a $1 \%$ hypertensive sodium chloride solution induced sputum is only detected in patients with severe asthma. ${ }^{9}$ The association of elevated levels of IL-8 with an increase in neutrophil count in induced sputum in patients with severe asthma is described by a number of researchers, explaining this by the presence of persistent viral infection in this category of patients. ${ }^{6,8}$ The data obtained by us correlate with the results of these studies and allow us to conclude that in the BA of severe course, without exacerbation, the content of IL-8 is not determined.

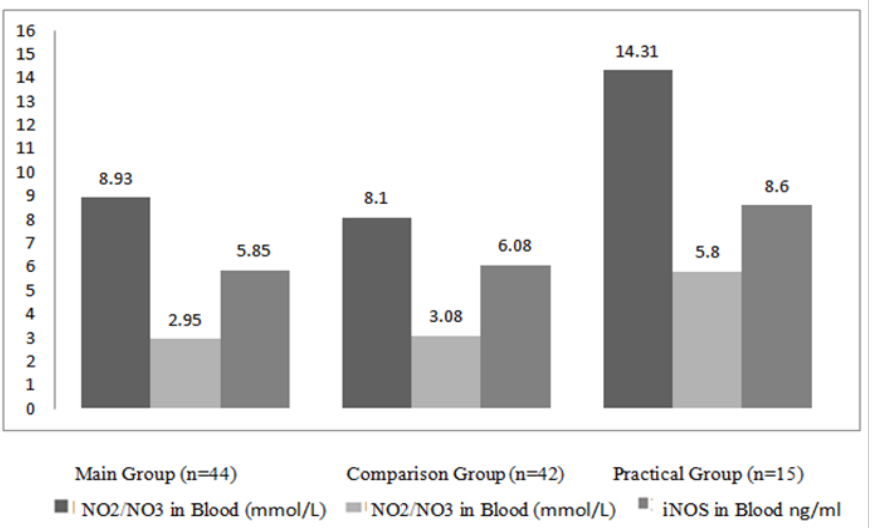

Figure I Initial data of indices of the endothelial system.

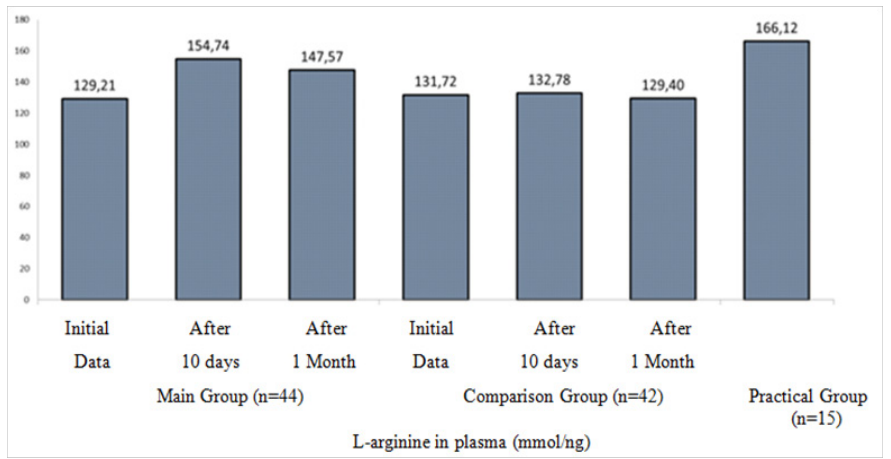

Figure 2 Indicators of L-arginine in the blood on the background of treatment.

When L-arginine was included in the treatment complex, along with the improvement in the clinical course of the disease, there was a significant $(\mathrm{p}<0.05)$ decrease in IL-4, IL-8 and TNF- $\alpha$ in the blood on standard therapy and L-arginine to the control period (10 days from the start of therapy) in the main group and continued to decrease when tested after 1 month. Changes in these parameters in the control group were less pronounced and were not reliable (Figure 3 ). When studying the content of IL-4 and TNF- $\alpha$ in the exhaled air condensate against the background of standard therapy and L-arginine after ten days, the indices decreased reliably $(\mathrm{p}<0.05)$ and continued to decrease after 1 month. Changes in these parameters in the comparison group (without the inclusion of L-arginine) were less pronounced and did not reach the confidence threshold.

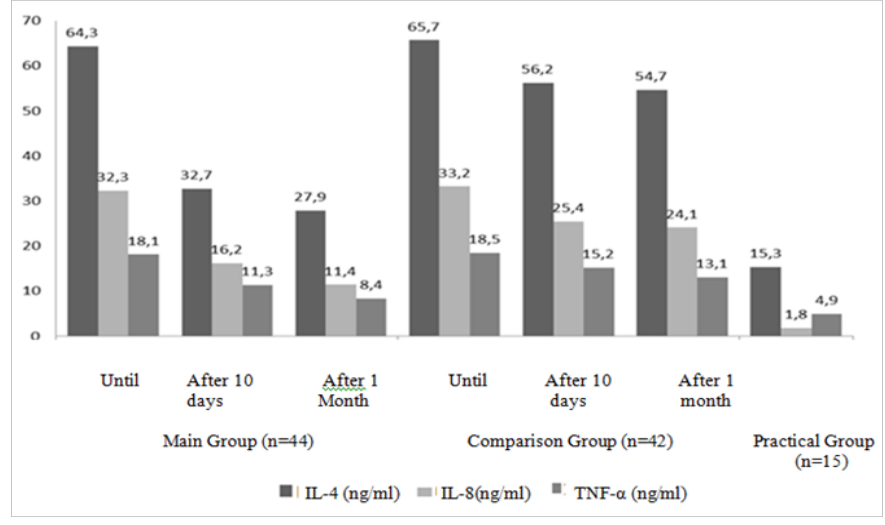

Figure 3 Dynamics of immunological parameters in the blood on the background of treatment.

The inclusion of L-arginine in the baseline therapy contributed to an earlier and significant decrease in the content of cytokines studied in the exhaled air condensate (Figure 4). The contents of interleukins in the exhaled air condensate after a ten-day treatment in the main group decreased almost to the conditional norm and continued to decrease after a month $(\mathrm{p}<0.01)$. Interleukin levels in the blood decreased, but did not reach the norm.

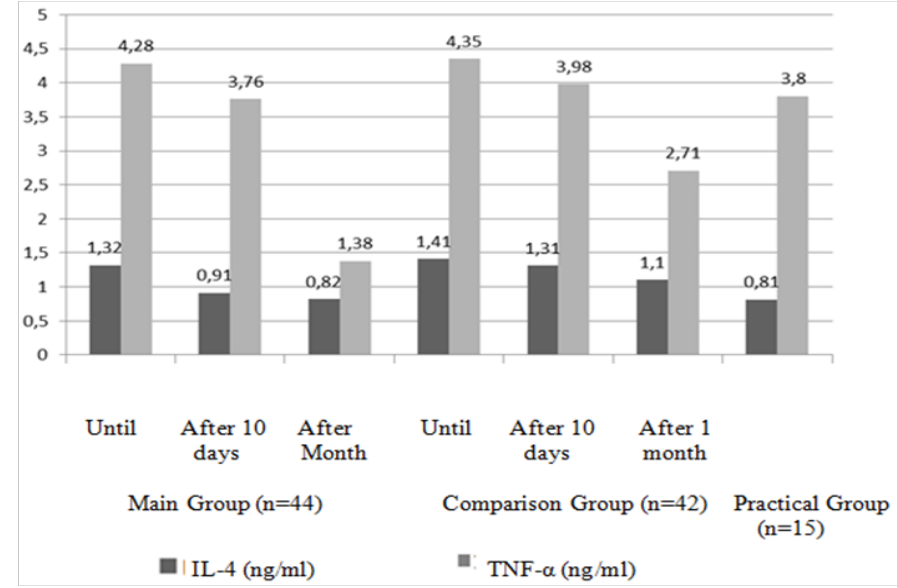

Figure 4 Dynamics of immunological parameters in KVV on the background of treatment.

\section{Indices of the endothelial system against therapy with inclusion}

L-arginine in the main group of patients showed a significant increase in the level of L-arginine and nitrate ions in the blood serum, as well as in the condensate of exhaled air. The increase in baseline in the comparison group was insignificant and was not reliable (Figure 2) (Figure 5). 


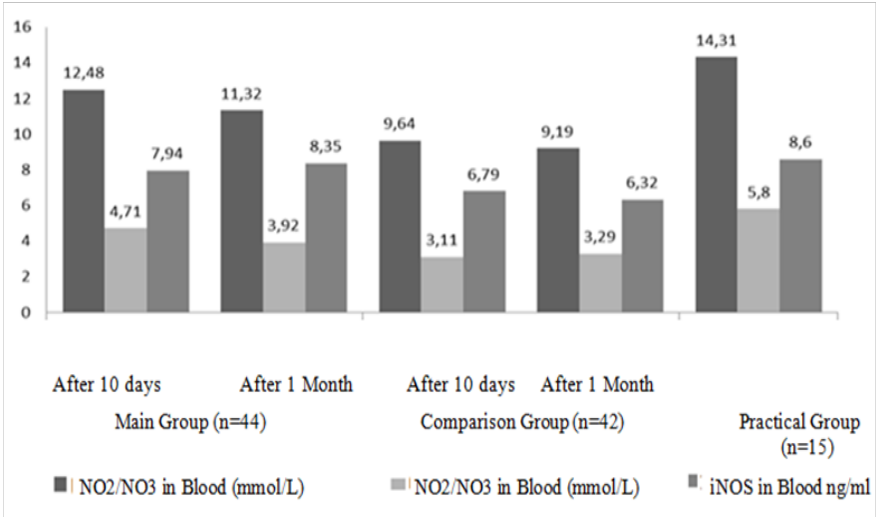

Figure $\mathbf{5}$ Indices of the endothelial system on the background of treatment.

\section{Discussion}

The content and ratio of cytokines in the blood serum reflect the nature of inflammation depending on the periods of exacerbation and remission and are not characterized by specificity. Changes in the content of cytokines in KVB reflect the activity of local inflammation in the respiratory tract in patients with asthma and are characterized by a positive dynamics under the influence of L-arginine. CABG patients with persistent asthma of moderate-to-moderate course are diagnosed with cytokines (IL-4 and TNF- $\alpha$ ). The study of cytokines in the exhaled air condensate makes it possible to assess the degree of inflammation, which allows us to clarify the further tactics of managing patients with asthma.

\section{Conclusion}

The study showed that with long-term bronchial asthma (average $16+4.5$ years) of mild and moderate persistent flow, endothelial dysfunction is formed with a decrease in the level of L-arginine in the plasma and a decrease in nitrate ions in the blood and in the condensate of exhaled air. In patients with prolonged course of persistent asthma of moderate severity, the use of a nitric oxide donor (Tivortin) in addition to basic therapy contributes to an earlier and reliable improvement of the immunological parameters studied. When L-arginine is used against the background of pathogenetic therapy, there is a positive dynamics of endothelial system indices, which is manifested by normalization of L-arginine content in blood serum and reduction of nitrate ions in blood serum and in the condensate of exhaled air in a short time. Received preliminary positive results dictate the need for further study of the mechanisms of L-arginine influence on the endothelial function in bronchial asthma and the possibility of its preventive use.

\section{Acknowledgements}

None.

\section{Conflict of interest}

The author declares no conflict of interest.

\section{References}

1. Chuchalin AG. Bronchial asthma and asthma-like conditions. Pulmonology. 2007;11:1-9.

2. Gozhenko AI, Kotyuzhinskaya SG, Kotyuzhinsky AI. Role of nitric oxide in the regulation of microcirculation and the aggregate state of blood. $\mathrm{Ukr}$ med almanac. 2000;1:13-17.

3. Kamilova UK, Abdullaeva CA. Study of indices of endothelial dysfunction and oxidative stress in patients with chronic heart failure. Eurasian Journal of Internal Medicine. 2014;(1):44-49.

4. Hodosh EM. Bronchial asthma: assessment of severity and optimization of therapeutic control. Health of Ukraine. 2005;1-2:10.

5. Zefirov AL, Haliullina RR, Anuchin AA, et al. Influence of endogenous nitric oxide on the function of the neuromuscular synapse. ROS fiziol IZM Sechenova. 2001;(4):499-5069.

6. Ghosh S, Erzurum SC. Nitric oxide metabolism in asthma pathophysiology. Biochim Biophys Acta. 2011;1810(11):1008-1016.

7. Silkoff PE, Robbins RA, Gaston B, et al. Endogenous nitric oxide in allergic airway disease. J Allergy Clin Immunol. 2000;105(3):438-448.

8. Nagaya N, Uematsu M, Oya H, et al. Short-term oral administration of L-arginine improves hemodynamics and exercise capacity in patients with precapillary pulmonary hypertension. Am J Respir Crit Care Med. 2001;163(4):887-891.

9. Vanin AF. Nitric oxide in biomedical research. Vestn RAMS. 2000;(4):35 .

10. Patent application for the device for collecting condensate of inspired air No. FAP20140050. 